\title{
PUZZLE-BASED LEARNING: AN APPROACH TO CREATIVITY, DESIGN THINKING \& PROBLEM SOLVING. IMPLICATIONS FOR ENGINEERING EDUCATION.
}

\author{
Stacy A. Costa \\ University of Toronto \\ stacy.costa@mail.utoronto.ca
}

\begin{abstract}
This paper will explore research practices already being conducted in various institutions, strengthening this paper's position that puzzle-based learning is a foundational methodology which assists newly admitted undergraduate engineering students, how to best approach critical problem solving. Furthermore, this paper will provide recommendations for an introductory protocol to introduce the incorporation of puzzle-based learning into a seminar-style course for First Year Introductory Engineering, and as a component of the engineering degree. The paper results in an exploratory analysis of what could be a starting place for future studies or classes to be conducted.
\end{abstract}

Keywords: Puzzle-Based Learning, Engineering Education, Creativity, Problem Solving.

\section{INTRODUCTION}

The human imagination and a search for order has been a part of humanity. For as long as problems have needed solutions, within the discipline of engineering, engineers have been trained, to design the best, most efficient solutions. When an engineer encounters a design or pedagogical dilemma, there is also an inner struggle they must face between order and chaos. A similar dilemma occurs when one is presented with trying to solve a puzzle. A puzzle can be comprised of various forms, logical, scientific, mathematical, mechanical, or verbal to name a few.

How can engineering education best decipher innovative practices in which engineering students can grasp design outcomes on project performances? How can higher education institutions train students, when we do not even know what the future professional workplace holds for these cohorts? Our students need to be prepared to be a part of an adaptive solving mindset to face challenges we cannot even begin to imagine.

\subsection{Puzzle Based Learning}

As per Falkner et al. (2012) [7], "Puzzle-based learning courses are not about presenting and discussing a variety of puzzles, but rather about presenting, discussing, and understanding problem-solving principles and some mathematical principles in the context of puzzles that serve as entertaining illustrations of the concepts presented." (p. 190).

Puzzle-based learning can allow students to become "knowledgeable entities" whom explore knowledge at a meta-cognitive level through the problem-solving format. As per Vygotsky (1982) [4] "the creative imagination is a result of the capacity of fantasizing situations." (p.7) When students engage in puzzle-based learning, they explore facets of a task and begin to formulate a deeper understanding of the function which can be elaborated in class.

Puzzle based learning must consist of problems which are challenging to solve, and must only consist of one correct solution, however can have different methods to solve. Puzzle-based learning should be a linguistically simple yet logically challenging problem. This pedagogy can be as useful as other educational techniques and approaches because it can challenge engineering students into an innate sense of creativity.

By crosscutting concepts that unify the study of engineering and through their common application across fields, (National Research Council 2012) [6], puzzles based learning allows for open-ended brainstorming, which connects creativity with disordered thinking and idea generating. The social rewards of co-creation amongst engineering students, leads to advantages of embodied and tactile forms of learning.

Puzzle based learning, can also been seen as a pedagogy in which becomes social, and allows for discussion across students, as well as a manner to encourage team work. While the discipline of engineering can be isolated, it is important that students collaborate 
and discuss their steps in working out solutions and how they approach the problem presented.

As per Falkner et al. (2010) [8], "The goal of puzzlebased learning is to lay a foundation for students to be effective problem solvers in the real world. At the highest level, problem-solving in the real-world calls into play three categories of skills: "dealing with the vagaries of uncertain and changing conditions; harnessing domainspecific knowledge and methods; and critical thinking and applying general problem-solving strategies." (p. 22) These categories of skill, present a modus operandi that allow for transferable skills to the workplace and are more crucial than more erudite aspects of education.

\subsection{Lateral Thinking}

Engineering Educators should want to foster an environment for first year engineering students to produce innovative thinking and emphasize idea-focusing. Some creative thinking can comprise of complex processes, that involves in perplexing problems that can seem unsolvable. By teaching methods for inventive thinking, this can help students to perform lateral thinking.

As per Danesi (2002) [5] Lateral thinking relies on reasoning that is not immediately obvious and encourages ideas that one may not immediately think of by relying on logic alone. As per Danesi (2002) [5], students must be taught these following steps when learning how to problem solve any task;

Challenge assumptions: question everything in view, and do not just fall back on accepted ways of thinking.

Focal Objects: pick a word a random within a problem and see what new thoughts, are related and can inspire other ideas.

Provide Provocation: deliberately set up a wild counterpart to the normally accepted idea, not as an end in itself but as a possible pathway to new ideas

Suspend judgement: many automatically stigmatize themselves and after a few seconds proclaim they do not know how to solve the problem or deem it as too difficult. Instead do not ruse to judge any ideas, however strange and difficult they may appear at first.

These steps assist in ways to approach problem solving across tasks, and ideas. We want the knowledge in which students acquire to link together and form connections in which develop enough experience in order to begin to solve a puzzle. Lateral thinking is embedded within all puzzle based learning and solving of any sort of puzzle. By having students incorporate lateral thinking into their repertoire of problem solving, it develops students to become more resilient.

\subsection{Concreteness Fading}

Fyfe et al (2012) [8], composed the concept of Concreteness Fading. This methodology consists of three main forms.

"the enactive stage - using a physical concrete model to represent the concept"

"the iconic stage - perceptual features unrelated to the concept are taken away and there are introductions of symbols and linkage of concrete and abstract objects."

"the symbolic stage - essentially an abstract model of the concept."

Fyfe et al (2012) [8] utilize this methodology in relation to teaching mathematics. With an instructor issuing a concrete object than slowing transition to students were found to not only achieve better marks, but also were found to perform and understand better than other students whom received a different method of instruction. This methodology can also be incorporated apart of how puzzlebased learning can take physical or mechanical puzzle problems and their representations to then become demonstrations to then lead into a better transitional of concrete to symbolic understanding. For some students, they may be concerned with just being given some symbolic representations due to lack of previous examples. While students can make connections across the symbolic as well as the enactive stage, students are able to understand and apply abstract concepts.

While concreteness fading has not been directly tested with puzzle-based learning, this paper takes the position that future studies can utilize concrete fading and attempt to create a study similar to Fyfe et al (2012) [8] with puzzle based learning and engineering problems. (Costa, Forthcoming) [9].

\subsection{How Puzzles Relate to Real Life Engineering Problems.}

Many engineering problems are puzzle like, as they are set up as logic problems. Pieces of the puzzle are provided to engineers in the form of user/customer requirements, technological constraints, professional or industrial codes, and market realities. The engineer must then craft a product or process that either meets all often-conflicting demands or else provides partial solutions.

With clear justification of the tradeoffs made when meeting all of the specifications is not possible. All engineers deal with puzzle like undertakings, computer engineers seem to face a much greater share of such problems

Parhami (2009) [2] notes that puzzle based learning is a task that directly relates to the job description of engineers. "Engineers must be constrained by user/customer requirements, technological, professional or industrial codes, and market realities. The engineer must then craft a 
product or process that either meets all of these demands." (p. 360)

\section{Puzzle-Based Learning as Pedagogy}

As puzzle-based learning is an experiential pedagogy, it seeks to provide a means to our pedagogical end. By allowing fluency, originality, and elaboration as a part of the cultivating discipline of engineering education, students will also gain skill sets which will allow maximum their learning performance and engineering careers.

As educators have been trying to find various methodological ways to induce creative thinking within the classroom, it seems to be a need to redefine creativity within the discipline of education. In an ever- accelerating capitalist society, that demands innovation, flexibility, and adaption especially from engineers, a global economic shift from production industries to knowledge circulation and curation, creativity is bound to shift from being a pursuit to a natural way of thinking and problem solving.

If learning persists within a classroom and the learning cannot be applied in any other context then what has happened within the classroom does not provide affordances. How are engineering classrooms then preparing students for long term learning post classroom. Puzzle-based learning can be a partial solution to this conundrum. As you we will see, the next section will present undergraduate case sties of how puzzle based learning has been utilized within engineering higher learning.

\section{Case Studies}

As Parhami (2008) [1], notes that Engineering freshman are not presented with practical problems, and can be less at ease with suffering with foundation problems. "Since it is difficult for freshman to grasp the underlying ideas of practicing engineers at the frontiers of research, puzzles can be the method to introduce the topic in a comprehensible way." (p. 263). Having puzzles as a part of a gateway course would allow for students to better retain information.

Parhami (2008) [1] introduced a computer engineering course in with she related popular mathematical and logical puzzles and students must then participate in formulating the solutions. The instructor takes $t$ up the solutions but then proceeds to demonstrate how the puzzles and the solution strategies are related to technical challenged in computer engineering. For example, utilizing
String Matching with Word Search, or Malfunctioning diagnosis with Logical Reasoning.

Another Case Study example is from Salcedo-Sanz et al (2007) [9] whom utilized Evolutionary Algorithms through Japanese Puzzle Nonograms. In their work, numbers are situated on a NxM grid, and certain blocks of cells must be filled in the grid in the order which they appear. In regard to this course, students participate in advanced encoding and must learn to construct a memetic algorithm.

In a third Case study, Marrick (2010) [3], the describes a formalized puzzle-based learning course in which introduced an introductory engineering computer programming course. As a compulsory course for all Bachelor or Engineering degrees at the University of New South wales. The author stated that "students learned an introductory knowledge of structure approach to problem solving. Puzzles were used to shift the focus towards a practical tool that can be used by engineers to problem solve." (p.677). The instructor utilized problems similar to those students could encounter, however had the problems and puzzles not be solely examples within a science of computing approach.

This mindset allows students to be able to problem solve and be prepared to encounter problems which may not be so clearly relatable but can help assist and understand similar steps in and familiarity across engineering environments they may encounter. This includes debugging, plotting, functions, testing, vectors, algebra and matrices. Puzzles were introduced with an interactive demonstration. One example being a simple physic model. By giving students time to work on this problem, students can then be encouraging to demonstrate several solutions and answers to the problems. Thus, having students engage deeply into the activity, entertain, and stimulate thought. Puzzles also were chosen by having a counterintuitive solution.

What these three cases studies have in common is the fact that each course does not just provide abstract thinking but relates abstract to concrete examples thus demonstrating Fyfe's et al (2012) [8] Concreteness Fading methodology. In addition, each course focused on attendance and participation as the stipulations for grading. All classes are on a pass or fail mark, and it is a mandatory course, in which has no more than thirty students participating at a time.

\section{Recommendations}

Puzzles demonstrate a method to apply student's understanding and competence, and building on concepts with previously taught concepts in order to accumulate difficulties and completing the problem.

Puzzle based learning should also allow for students to utilize abstract reasoning critical thinking and logical 
reasoning, as categories of abstract models, and become transferable to dealing with uncertainty, working in teams, identifying questions, all tasks which occur in the real world and are transferable skills. Canadian Higher institutions should begin by looking at concepts which are difficult, and highly abstract, in which can best be related into problem solving tasks, as well as understanding how students can better utilize teamwork to incorporate insight thinking at the individual and group level. Furthermore, it is important that instructors also develop within their course and emphasize the mathematical, reasoning and proof with increasing difficulty as the course continues over time.

As Falkner (2012) [7] notes that engineering industries values problem solvers, thus applying their skills in any similar domain can also be an asset in which can assist engineers to be job ready for a multitude of positions, and recognize that they can accomplish more in the industry as a resilient engineering graduate.

\section{CONCLUSION}

Within this paper has been presented brief literature examples of current puzzle based seminar style classes which have engaged in undergraduate engineering programs. These examples demonstrate successful implementation of puzzle based learning as methodology of innovative thinking. While these courses are still in their infancy, and there needs to be more evaluation and longterm study, we can learn from these to better prepare and plan courses for engineering undergraduate students. By creating a curriculum that embodies some of these recommendations, as well as following in the successful case studies listed in the paper, this paper hopes to inspire future classes in higher education regarding problem solving and puzzle based learning, as well as research being done in this area.

While minimal details have been included, this paper can be a starting place for future protocol in Canadian universities to consider a puzzle based learning course. By having engineering educators understand and embrace puzzles within their coursework, puzzles are not to overshadow the important curriculum guidelines in place for engineers but instead to be put in conjunction with current guidelines.

Solving puzzles entails the ability to compare hidden information in a puzzle with information already in memory, and, more importantly, the ability to combine the information to form novel information and ideas. The thinking involved in solving puzzles can thus be characterized as a blend of imaginative association and memory. It is this blend, I would claim, that leads us to literally see the pattern or twist that a puzzle conceals. It is what provokes an Aha! effect.
Finally, through puzzle based learning, it is meant to discover creative thinking, and idea-generating solutions. Thinking can be a complex process that involves moments of order and disorder. Such inventive thinking

\section{Acknowledgements}

Thank You to Dr. Greg Evans, and Dr. Deborah Tihanyi for their feedback in the presentation version of this paper, during our Class seminar. Your knowledge, questions as well as theoretical dissemination of knowledge has been valuable. I am grateful for your endless suggestions and through provoking comments in every Engineering Education class, that has led me to this. As well, thank you to Dr. Marcel Danesi whom introduced me to this field over seven years ago, and has continued to inspire the creative thinking and forward push in the field. I am forever indebted to you.

\section{References}

[1] Behrooz Parhami. "A Puzzle-Based Seminar for Computer Engineering Freshman," Computer Science Education. Vol. 18, no. 4, pp. 261-277, 2008.

[2] Behrooz Parhami. "Motivating Computer Engineering Freshman Through Mathematical and Logical Puzzles," IEEE Transactions on Education. Vol 52, no. 3, pp 360-364, 2009

[3] Kathryn E. Marrick. "An Empirical Evaluation of PuzzleBased Learning as an Interest Approach for Teaching Introductory Computer Science," IEEE Transactions on Education, Vol 52, no. 4, pp. 677-680, 2010.

[4] Lev Vygotsky. Imagination and Creativity in Childhood. New York, NY: Plenum Press, 1982 ( $1^{\text {st }}$ ed.) 97 pp.

[5] Marcel Danesi. The Puzzle Instinct. The Meaning of Puzzles in Human Life. Bloomington, Indiana University Press, $2002\left(1^{\text {st }}\right.$ ed.) $263 \mathrm{pp}$.

[6] National Research Council. A Framework for K-12 science education: Practices, crosscutting concepts, and core ideas. Washington, DC: National Academies Press, 2012, 5 pp.

[7] Nickolas Falkner, Raja Sooriamurthi, \& Zbigniew Michalewicz. "Teaching Puzzle-Based Learning: Development of Transferable Skills" Teaching Mathematics and Computing Science. Vol. 10, no 2, pp. 245-248, 2012.

[8] Nickolas Falkner, Raja Sooriamurthi, \& Zbigniew Michalewicz. "Puzzle-based learning for engineering and computer science." Computer, IEEE Computer Society, Vol 43, no. 4, pp 20-28, 2010.

[8] Nicole McNeil \& Emily Fyfe. "Concreteness Fading promotes transfer of mathematical knowledge." Learning and Instruction. Vol 22, no 1, pp 440-448, 2012. 
[9] Sancho Salcedo-Sanz, Jose A. Portilla-Figuera, Emilio G. Ortiz-Garcia, Angel M. Perez-Bellido and Xin Yao. "Teaching Advanced Features of Evolutionary Algorithms Using Japanese Puzzles." IEEE Transactions on Education. Vol, 50 no 2, pp 151-156, 2007.
[10] Stacy Costa. "Puzzle Based Learning and Concrete Fadedness.” Knowledge Building Summer Institute Proceedings. Forthcoming. 